\title{
La giovinezza tenace. I luoghi e le parole di Giacomo Ulivi
}

Riscoprire la storia e le ragioni delle scelte di un ragazzo di 19 anni che aderì alla Resistenza e cadde combattendo per la libertà: questo era l'obiettivo del laboratorio didattico proposto ad alcuni liceali. Giacomo Ulivi è un esempio di tenacia, forza e coscienza civile. Attraverso le foto, le sue lettere e i documenti gli studenti hanno saputo incontrare un loro "coetaneo di tanti anni fa".

The unfolding of a 19 year old's life who decided to combat for freedom e died fighting for the italian resistance movement during German occupation in World War II. An example of tenaciousness, strength and social consciousness. This was the reason of the educational workshop for high school schoolers achieved using Ulivi's private documents, photos and letters. In this way the students met someone of their "own age" who lived many years back.

«No, non dite di essere scoraggiati, di non volerne più sapere. Pensate che tutto è successo perché non ne avete più voluto sapere!» [Lettere di Giacomo Ulivi 1974]. Sono le parole più conosciute di Giacomo Ulivi, che lui stesso scrisse nei mesi dell'esilio modenese rivolgendosi a tutti gli "amici". Queste poche righe, ormai note, sono state riprese più volte anche da autorità come il Presidente della Repubblica Giorgio Napolitano. Tenendo ben presente la loro importanza, il Liceo scientifico intitolato proprio a Giacomo Ulivi e l'Istituto storico della Resistenza e dell'età contemporanea di Parma hanno voluto costruire un progetto sulla figura del giovane partigiano, per trasmettere agli studenti di oggi ideali di impegno civile che sembrano essersi persi nel tempo.

Oltre all'Isrec Parma e al Liceo “G. Ulivi” l'iniziativa si è avvalsa della collaborazione e del sostegno delle associazioni partigiane Alpi, Anpi e Apc, dell'Istituto 
storico della Resistenza e dell'età contemporanea di Modena, di Coop Consumatori Nordest, del Gruppo Spaggiari e ha beneficiato del patrocinio di Comune e Provincia di Parma.

Il progetto, i cui esiti sono una mostra (aperta al pubblico dal 5 aprile al 31 maggio 2014) e una pubblicazione - la cui uscita è prevista per il novembre prossimo - ha avuto come punto di partenza la realizzazione di un laboratorio storicodidattico, ideato e condotto da Michela Cerocchi (Isrec Parma) in collaborazione con gli insegnanti del Liceo scientifico.

Nel corso dei mesi in cui si è svolta l'attività formativa è stata indagata la vita di Giacomo Ulivi sia nei suoi aspetti privati, attraverso le numerose foto di famiglia contenute nell'archivio del cugino Fausto Fornari, sia in quelli pubblici legati all'impegno resistente.

A seguire con entusiasmo e impegno il progetto sono stati una trentina di ragazzi provenienti da classi diverse, dalla seconda alla quinta liceo. L'obiettivo dell'intero progetto è stato quello di far riaffiorare la storia del giovane Giacomo, conoscendolo meglio e andando oltre quelle poche righe ormai monumentalizzate. Per fare ciò i ragazzi hanno avuto l'occasione di indagare la vita, i pensieri e le parole di quel loro coetaneo che con coraggio mise a rischio e perse la propria vita per la Resistenza.

Il percorso didattico si è districato tra i luoghi fondamentali per la crescita e la maturazione di Ulivi (la casa di famiglia, la scuola, Parma e poi Modena) e le parole: le sue, quelle delle lettere al compagno di scuola Ennio Loyola e quelle scritte da Modena alla madre e all'amico W., Vittorio Pellegri. Attraverso i luoghi e le parole dunque - parafrasando il sottotitolo assegnato al progetto - gli studenti si sono avvicinati a Giacomo, riconoscendolo come un loro "coetaneo di tanti anni fa". Proprio questa vicinanza anagrafica ha fatto sì che l'accostamento sia avvenuto con naturalezza ed entusiasmo. I ragazzi hanno riconosciuto in lui non un eroe freddo e lontano, ma un giovane vivo, appassionato e vero. Terminato il percorso didattico il lavoro non si è fermato ed è confluito in una mostra dal titolo $L a$

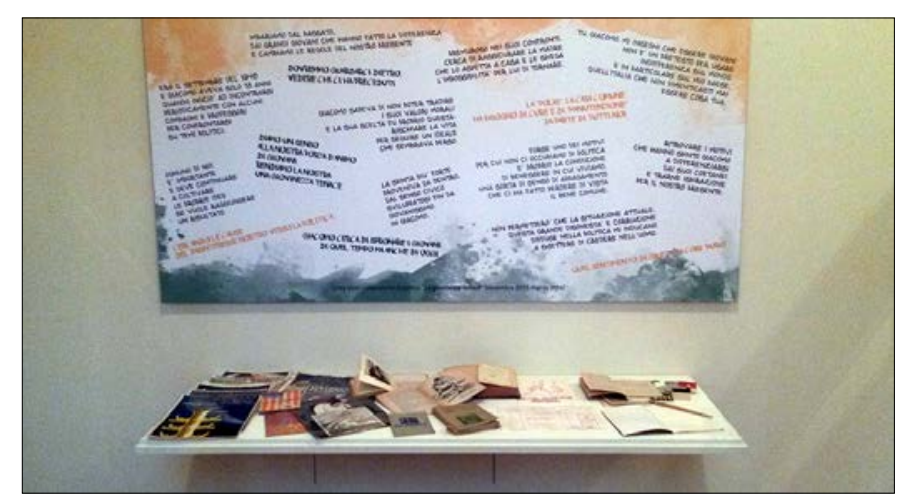

Pannello della mostra, che raccoglie alcune delle riflessioni scritte dagli studenti durante i laboratori 
giovinezza tenace. I luoghi e le parole di Giacomo Ulivi, esposta presso la Casa della Musica di Parma, nella quale documenti, foto, lettere, pagelle si sono legati ai pensieri e alle riflessioni scritti dagli alunni durante i laboratori. Si è venuto così a creare un intreccio tra passato e presente molto significativo.

È stato l'album di famiglia a favorire il primo "incontro" degli studenti con Giacomo; le tante fotografie, custodite negli anni dall'attento cugino Fausto Fornari, hanno mostrato la vita quotidiana di una famiglia benestante negli anni Trenta e Quaranta. Attraverso gli scatti è stato possibile seguire Giacomo Ulivi nei suoi primi anni di vita, prima nella villa di Baccanelli, in provincia di Parma, con i nonni materni e la zia Teresa, poi a Bruxelles, dove la famiglia si era trasferita per seguire gli impegni lavorativi del padre Giulio, inventore e scienziato. Infine di nuovo a Parma con la madre Maria Luisa (il padre per lavoro rimase a Bruxelles) - un periodo documentato anche dalle foto di classe delle elementari e da quelle del gruppo della Cresima. Attimi semplici di una famiglia facoltosa che, anche negli anni duri della dittatura fascista, voleva e poteva permettersi una vita normale e agiata. In molte foto Giacomo è ripreso con sua madre e i parenti al mare, in villeggiatura sulle coste liguri e adriatiche. Il rapporto del giovane Ulivi con la madre era molto stretto sia perché, vista la lontananza del padre, Maria Luisa era il suo principale punto di riferimento, sia perché Giacomo era il terzo figlio della coppia dopo due bimbi morti nei primi mesi di vita.

Uno dei luoghi più importanti per la crescita di Giacomo, su cui gli studenti si sono soffermati a lungo, è la scuola. Nel 1935 Maria Luisa iscrive il proprio figlio al Convitto "Maria Luigia"; tra i banchi di scuola Ulivi si sente a suo agio, ama conoscere cose nuove e la sua curiosità e attenzione non hanno mai fine. Punti di riferimento fondamentali soprattutto negli anni del liceo classico sono i professori che, nonostante i divieti e i controlli delle autorità fasciste, cercano di trasmettere agli studenti un senso critico della realtà, oltre ad offrirgli strumenti per leggere in modo chiaro il presente. Tra gli insegnanti di Giacomo troviamo nomi noti nell'ambiente antifascista parmigiano come don Giuseppe Cavalli, Tito De Stefano e Italo Petrolini. Un altro suo professore, il poeta Attilio Bertolucci, ha ricordato così quegli anni:

Erano gli anni 1940-1941, anni di angoscia e di speranza indicibile per il nostro piccolo gruppo di professori in età di chiamata alle armi, vergognosamente antifascisti, anglofili, incapaci di stare zitti, eccetera. Come potevamo non contagiare i più intelligenti, e i più puri, degli allievi che ci stavano intorno ore e ore, ogni giorno, e avevano soltanto pochi anni meno di noi, e dimostravano visibilmente una così urgente volontà di conoscere e di vivere? Ognuno di noi faceva la sua parte, devo dire forse con più incoscienza che lucido disprezzo del pericolo, e ognuno 
secondo il suo temperamento e il suo carattere [Alberghi 1976, 57].

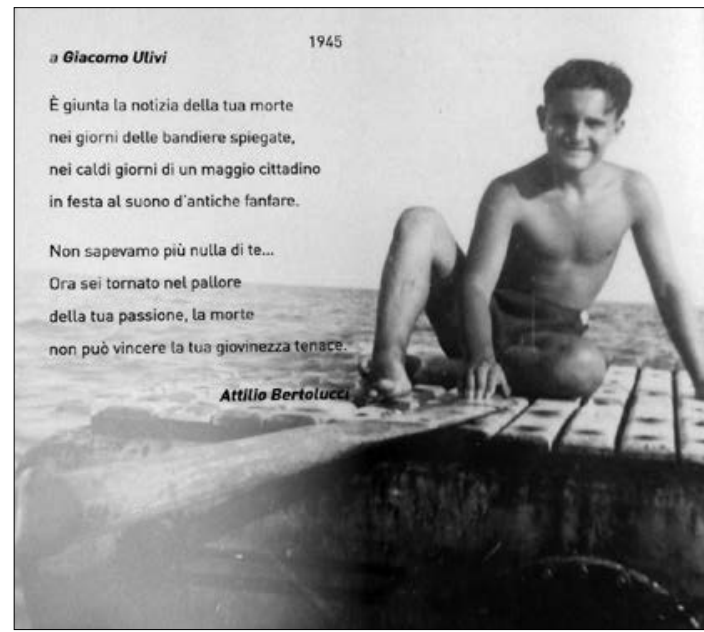

Immagine di Giacomo Ulivi in barca e poesia di Attilio Bertolucci a lui dedicata
Giacomo viene ben presto contagiato da quel sentimento antifascista e non solo grazie ai professori, ma anche grazie alla figura maschile più vicina a lui, lo zio Giulio Fornari. Con lui il giovane Ulivi passava ore a parlare dei temi più attuali, mostrando una maturità e un disincanto eccezionali.

Gli studenti hanno avuto così l'opportunità di riflettere sul ruolo dell'educazione sotto un regime dittatoriale e di spostare l'attenzione anche sul loro presente.

A scuola Giacomo si legò molto ad alcuni compagni, come Ennio Loyola, che dovette però allontanarsi da Parma per tornare a Roma insieme alla famiglia. Nei primi anni Quaranta i due si scrissero alcune lettere; ed è proprio grazie a quegli scritti che gli studenti hanno potuto scoprire anche il Giacomo "ragazzo", divertente e ironico, giovane come loro. Con queste parole, ad esempio, raccontava all'amico il suo esame di maturità:

Ho tanto ritardato a scriverti, perché, contrariamente alle previsioni ed alle decisioni, ho dato l'esame.

Mi sono deciso molto tardi, e quindi non ho avuto il tempo di prepararmi come avrei voluto: ciò nonostante è andato bene. [...] Attendo con ansia una tua lettera, che mi racconti tutto ciò che fai. Se non fosse troppo lungo, ti racconterei per filo e per segno le mie peripezie di maturando: specialmente l'esame di matematica, che all'ultimo momento mi hanno consigliato di tentare, è stato un interessante spettacolo. Pensa che dopo aver risolto (senza sapere con eccessiva precisione cosa sia la trigonometria) un elegante problemino a basi di "tangenti" (?), aiutato da un posteriore misurabile in ettari, ho dovuto confessare che le divisioni con due cifre non facevano parte del mio repertorio... [Lettere di Giacomo Ulivi 1974, 24].

Oltre all'ambiente scolastico gli studenti hanno iniziato a conoscere la Resistenza di Giacomo.

Ulivi era dotato di un antifascismo "intellettuale", nato e sviluppatosi attraverso 
l'educazione e la cultura, e di un senso critico che lo portò presto a un'importante convinzione: una società che vietava la libertà di parola, di stampa e di associazione, che entrava nel privato, che imponeva la propria cultura, $\mathrm{i}$ propri libri e le proprie idee, non poteva essere una società giusta. Uno dei primi atti resistenti di Giacomo fu la messa in scena, in modo ironico, ma estremamente intelligente, della liberazione di Benito Mussolini dalla prigione del Gran Sasso. Giacomo, insieme al cugino e ad altri ragazzi, realizzò un fotoromanzo, con immagini e didascalie che spiegavano azione dopo azione l'impresa dei paracadutisti tedeschi. Gli studenti ne hanno studiato il linguaggio, sarcasticamente ricalcato sulla retorica del regime, e ne hanno potuto osservare le fotografie. Quello che a prima vista sembrava solo un gioco tra ragazzi in realtà non era un semplice passatempo, ma un'azione consapevole e disincantata, un'operazione di Resistenza in senso proprio.

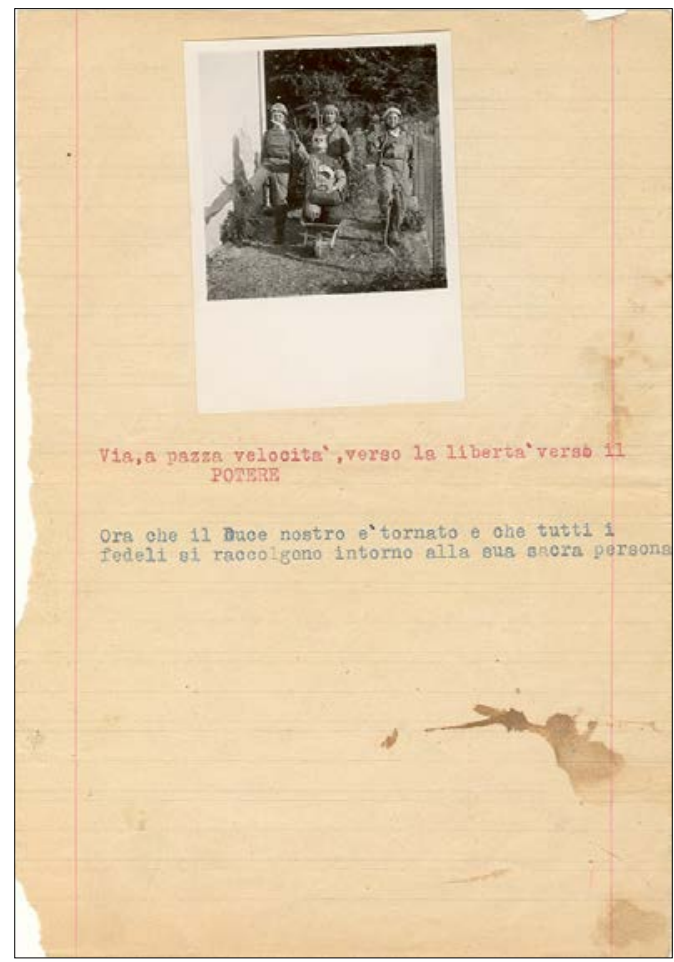

Foglio della parodia dell'Operazione Quercia, messa in scena da Giacomo Ulivi, Fausto Fornari e altri amici nella villa di Ozzano Taro - settembre 1943

Successivamente i ragazzi hanno seguito Giacomo nel suo avvicinarsi al movimento clandestino di Parma. Già da qualche mese prima dell'annuncio dell'armistizio Ulivi si riuniva con ex compagni di classe e professori per discutere delle vicende belliche e dei modi per agire contro il regime fascista. Dopo l'8 settembre 1943, tuttavia, non gli bastarono più le riunioni e le discussioni, e si decise per l'azione.

Non conosciamo con certezza tutti i legami che Ulivi costruì in città ma sappiamo che il giovane studente si impegnò attivamente nella diffusione di stampa clandestina, volantini, giornali, manifesti. La rete di conoscenze tra ex-alunni e professori non passò inosservata agli occhi attenti dei fascisti che cercavano in ogni modo di reprimere il movimento resistente in città. Partì così un'ondata di 
arresti che raggiunse anche Giacomo, il quale, una volta portato nella caserma della Guardia nazionale repubblicana, riuscì però a fuggire e grazie a contatti e amicizie raggiunse Modena.

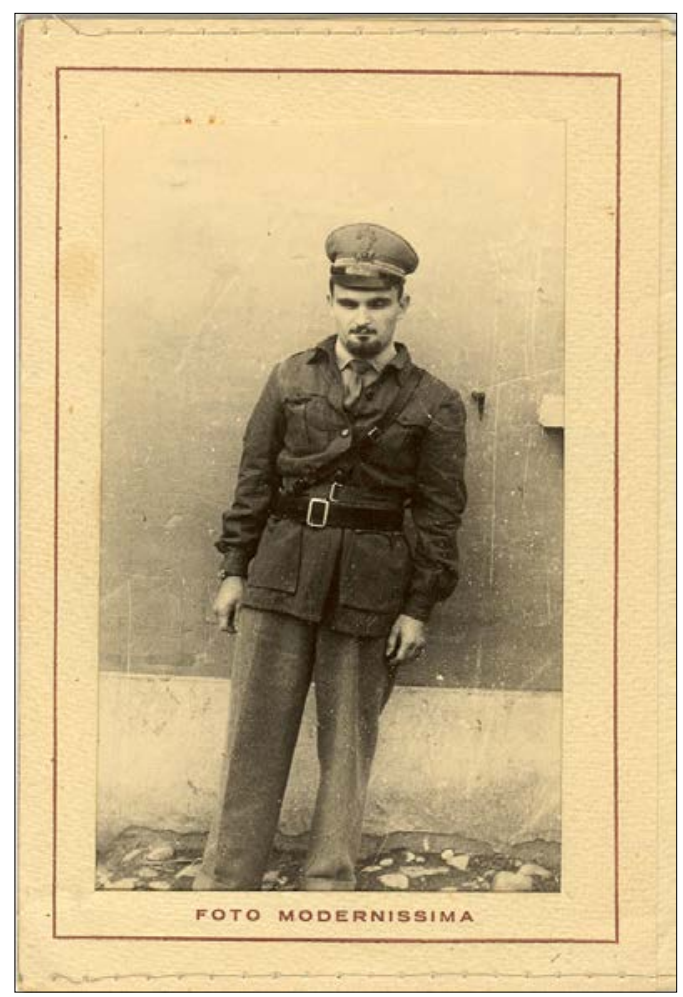

Giacomo Ulivi in divisa, probabilmente un travestimento per passare inosservato tra le vie di Modena
Gli studenti del Liceo scientifico "G. Ulivi" hanno seguito Giacomo anche in quel luogo, andando a visitare la città, la casa dove venne ospitato e le vie che percorse con la paura di cadere ancora in mani nemiche. Poche sono le informazioni sulle attività modenesi del giovane parmigiano. $\mathrm{Si}$ sa che venne ospitato nella casa del maresciallo Alessandro Bassi, interprete per l'esercito tedesco nel Comando militare provinciale repubblicano, ma in realtà spia della Resistenza. Attraverso quel contatto Ulivi riuscì a inserirsi nel movimento clandestino modenese.

La vita in città era molto difficile: per non farsi riconoscere Giacomo si fece crescere la barba e solo "protetto" dai documenti falsi ottenuti dal maresciallo Bassi pote-

va muoversi per le vie di Modena. La voglia di lasciare la città e salire ai monti, di fare finalmente il suo «entusiasmante dovere» era forte [Lettere di Giacomo Ulivi 1974, 36]. Dalle lettere alla madre della metà del settembre 1944 possiamo intuire la sua volontà di prepararsi a quella decisione.

Se la posizione si stabilizza - penso sempre al peggio - avrei bisogno di quel vestito pesante spinato, col quale partii. Infatti quello grigio è troppo bello, e quello nocciuola, come sai, è sempre indecente, e, profilandosi mesi freddi e senza molto riscaldamento, impossibile. Se puoi, anche un paio o due di calzettoni. Non so se queste tre cose sono maturate! Ma se non puoi così è lo stesso. Tutto questo lo dico, nel caso che la situazione, cristallizzandosi, conduca a uno «sventramento», il che credo non probabile; ma le misure vanno sempre prese assai larghe! [Lettere di Giacomo Ulivi 1974, 73-74]. 
Scarponi, calze pesanti, vestiti di lana, tutto l'occorrente per affrontare l'inverno sui monti. Sfortunatamente Giacomo non riuscì mai a raggiungere gli altri gruppi partigiani presenti sulle alture modenesi: il 30 ottobre 1944 alcuni uomini della Brigata nera lo fermarono e lo arrestarono. Alcuni giorni dopo, precisamente il 10 novembre 1944, il giovane parmigiano venne scelto, insieme ad altri due prigionieri, per dimostrare la forza fascista di fronte ad un'azione partigiana. I tre uomini furono portati nella piazza principale della città e fucilati per rappresaglia. A Modena, dove Ulivi è caduto, si è concluso anche il percorso seguito dagli studenti nel corso del laboratorio. Non si sono fermati però i loro pensieri e la voglia di capire ancora meglio l'esempio del giovane Giacomo. Hanno continuato a scrivere e a riflettere:

Nonostante la sua giovane età, non smise mai di lottare e non tornò mai indietro, fino a che la battaglia non trovò la fine con la sua fucilazione in Piazza Grande a Modena. Giacomo rappresenta per noi un modello al quale qualsiasi ragazzo anche oggi dovrebbe ispirarsi: senso del dovere e responsabilità, quotidianità e giovinezza, in perfetta unione tra loro. Rebecca Cirri

E così la consolidata ribellione interiore contro l'oppressione dittatoriale rompe le catene e si fa azione, vero e proprio antifascismo: dalla scambio di opinioni con i suoi mentori, passa alla satira politica e assieme agli amici e al cugino Fausto si costruisce un'acuta forma di Resistenza morale. Nel ' 43 farà una scelta radicale, inevitabile per una mente conscia del presente e animata del suo stesso ardore, aderendo ai movimenti organizzati di Liberazione.

$\mathrm{Tu}$ Giacomo mi insegni che essere giovani non è un pretesto per usare indifferenza sul mondo, e in particolare sul mio paese, quell'Italia che non dimenticasti mai essere cosa tua. Oggi mi suggerisci di dare sfogo a tutta la carica dei miei 19 anni nell'eroico esercizio della cittadinanza. Fabio Ricchieri

La tua generazione ha lottato per la libertà, ha vissuto l'esperienza di un regime totalitario che ha negato le libertà fondamentali. Voi avete lottato per riaffermare quelle libertà di cui oggi godiamo a pieno. Tuttavia è cresciuto sempre più fino ad oggi un sentimento di lontananza dalla vita politica, mentre credo che il dono più grande che ci avete lasciato sia la partecipazione come espressione più grande della democrazia. Virginia Accolli 
Impariamo dal passato, dai grandi giovani che hanno fatto la differenza e cambiamo le regole del nostro presente perché i nostri mezzi rispetto a quelli che possedeva Giacomo Ulivi per cambiare le cose sono uguali se non superiori. Diamo un senso alla nostra forza d'animo di giovani, rendiamo la nostra una giovinezza tenace. Giulio Ceresini

I ragazzi hanno accolto la storia di Giacomo Ulivi e l'hanno fatta propria; si sono fatti portavoce dell'esperienza del giovane partigiano concretamente, facendo da guide alle tante classi di scuole secondarie di primo e secondo grado che hanno fatto visita alla mostra. Comprensione, emozione e trasmissione sono le parole che hanno accompagnato gli studenti in questo viaggio tra $\mathrm{i}$ documenti, le foto e la vita di un «giovane tenace».

\section{Bibliografia}

Lettere di Giacomo Ulivi 1974, Modena: Istituto storico della Resistenza

Alberghi P. 1976, Giacomo Ulivi e la Resistenza a Modena e Parma, Modena: Teic

\section{Risorse}

Liceo scientifico "G. Ulivi”

Parma, http://www.liceoulivi.it/pvw/app/PRLS0002/pvw_sito.php?sede codice $=$ PRLS0002\& from $=-1$ \&page $=277$

Biografia, Sito Anpi nazionale http://www.anpi.it/donne-e-uomini/giacomo-ulivi

Convitto nazionale "Maria Luigia", Parma http://www.marialuigia.eu/ulivi.asp

Ultime lettere di condannati a morte e di deportati della Resistenza italiana, Insmli http://www.ultimelettere.it/?page_id $=35 \&$ ricerca $=153$ 